\title{
Individualized Cancer Chemotherapy by Detecting Cancer Biomarkers
}

\author{
Da-Yong $\mathrm{Lu}^{1 *}$, Ting Ren $\mathrm{Lu}^{2}$ and Shan $\mathrm{CaO}^{3}$
}

${ }^{1}$ School of Life Sciences, Shanghai University, Shanghai 200444, PR China

${ }^{2}$ College of Science, Shanghai University, Shanghai 200444, PR China

${ }^{3}$ Zhong-Guo High School, Xu-Hui District, Shanghai, PR China

\begin{abstract}
A major obstacle to control cancer growth and metastases in patients is the widespread inappropriate use of anticancer drugs. Individualized cancer chemotherapy (ICC) has been attracting increasing attentions in medical circle and will continue to show beneficial outcome in the future. This review addresses this issue by discussing one specific strategy of ICC, biomarker-oriented therapy. Many longstanding key debates and future trends are discussed.
\end{abstract}

Keywords: Individualized cancer chemotherapy; Anti-cancer drugs; Bioinformatics; Cancer treatment; Tumor bioinformatics; Drug selection; Omics

\section{Introduction}

A major obstacle to control cancer growth and metastases in patients is the widespread inappropriate use of anti-cancer drugs. As increasing numbers and types of anti-cancer drugs are developed, clinicians become more and more likely to misuse them in their practice. The norm is to chemotherapy that is "randomized" or "standard" in relation to the target organs or tumor origins. Since tumors are originated from a wide variety of different phenotypic or genetic abnormal tissues, these different tumor tissues are sensitive to different anti-cancer drugs. Thus, most cancer patients are unsuited to the use of such "uniform" or "standardized" chemotherapy. As no single drug or combination has so far been found that is optimal for cancer of all origins, developing a good and clinically practical drug selection system is no less important than the discovery of new drugs. Researchers have now begun to focus on "individualized cancer chemotherapy" (ICC), which improves therapeutic quality by selecting and prescribing well-matched drugs and avoid ineffective ones [1].

\section{Bioinformatics for Individualized Cancer Chemother- apy}

Various molecules have been widely reported to have diagnostic and/or prognostic values in cancer patients. Such molecules range from immunoregulatory and inflammatory factors (interleukins and cytokines) and signal transduction regulators (tyrosine kinase, cycloxygenase-2, MAPK, etc) to factors related to tumor pathology (metastases, angiogenesis and apoptosis) such as vascular epithelial growth factor and its receptor (VEGF and VEGFR), EGFR and fibrinogen. These pathogenic markers in tumors are specific targets for drug antagonism or disruption [1-3]. With the increasing production of highly selective drugs (cytostatic anti-cancer drugs), cancer patients can be treated with targeted agents chosen by reference only to pathological data without drug sensitivity tests. Successful example can be seen in fluoro-pyrimidine based chemotherapy for metastatic colon cancers over expressing dihydropyrimidine dehydrogenase and thymidylate synthase [4] and treatment of the deadliest lung cancers resulting from epidermal growth factor receptor mutation with monoclonal antibody, Iressa [5].

In the tumor marker detections, bioinformatics have been the best suited methods to identify tumorigenic initiators and promoters, and to further decide which cytostatic anti-cancer drugs may possibly target. Since tumors are progressive conditions [6-7] that may result in more than a hundred genetic factor changes in a single cell [8-9], it demands high-throughput methods to simultaneously identify or pinpoint 3-digit numbers genetic abnormalities from 40-50 currently known cancer biomarkers in each tumor origin in future.

\section{Introduction of Methods}

Cancers possess different etiological basis with a same pathological characteristic of unlimited growths. They result from genetic malfunctions and molecular disturbances. More than a thousand types of genetic abnormalities can cause about one hundred different tumor types and the different genetic abnormalities must be treated or rectified by more than one hundred different anti-cancer drugs or even more. It means that responses to same anti-cancer drugs may be various from patient to patients. ICC is the way to do this.

\section{Prediction of drug responses by detecting tumor biomarkers}

Bioinformatics is a genomics-based approach and may possibly be the greatest varieties of techniques for analyzing genetic or genomic abnormalities of DNA, RNA, proteins and glyco-ligands in tumors. In the earliest era of bioinformatics development, clinical cancer practices were to predict patient's prognosis [10] or classifications of tumor origins [11]. Over-expressions of many oncogenes or biomarkers, such as HER2 in women or gastric cancer, will show poor prognosis of patients if a conventional therapy is used.

Presently, the best way of bioinformatics for the prediction of anticancer drug response is to decide monotherapy (treatment of cancer patients with monoclonal antibodies). If a tumorigenic biomarker has been detected in a remarkable higher level, it is reasonable to assume that monoclonal antibodies of specifically targeting this tumorigenic biomarker will be effective. Much success and numerous reports have been addressed this issue [12-15]. But monoclonal antibodies are very expensive. Thousands USD of drug expense can be only used in a single therapeutic cycle. Usually only a few months survival benefits can be

*Corresponding author: Dr. Da-Yong Lu, School of Life Sciences, Shanghai University, Shanghai 200444, PR China, E-mail: ludayong@sh163.net

Received September 18, 2012; Accepted September 18, 2012; Published September 25, 2012

Citation: Lu DY, Lu TR, Cao S (2012) Individualized Cancer Chemotherapy by Detecting Cancer Biomarkers. Metabolomics 2:e121. doi:10.4172/2153$0769.1000 \mathrm{e} 121$

Copyright: (c) 2012 Lu DY, et al. This is an open-access article distributed under the terms of the Creative Commons Attribution License, which permits unrestricted use, distribution, and reproduction in any medium, provided the original author and source are credited. 
expected. Also, many authors have reported that there is no survival benefit in patients after monotherapy [16-17]. We hypothesize herein whether monotherapy or RNAi agents targeted at original tumorigenic molecules might decide the effectiveness of following therapy. For example, treatment targeting chromosome translocations in childhood leukemia will improve the treatment outcome of patients with chromosome translocations, therapeutic benefits are improved from previous cure rate of $25 \%$ to now $80 \%$. So the bioinformatics detections is an important means to find out the most original and causative biomarkers in an individualized chemotherapy. In order to reach this goal, much more basic and clinical investigations are needed to build good routines and paradigms for clinical practitioners to follow. These routines and paradigms need to be easy to handle, less expensive, highthroughput, and as effective as possible. Currently, bioinformatics techniques have numerous selections and no single technique has been much advantageous over the rest of methods. We think some wellorganized international committee should be encouraged to work out with this issue in future. Some standard chips or techniques need to be designed and uniformed by some big manufacturers to safeguard the quality and price of detections.

\section{ICC targeting tumor metastases}

More than $90 \%$ cancer deaths are caused by cancer metastasis. As cancer metastasis is the main cause of human deaths, we shall pay more attentions on it. Currently, treatment and chemotherapy are focused on primary tumors rather than metastatic processes. Anti-metastatic drugs are often used as assistant therapy. So cancer patient's survivals have been improved very little. To change this mindset, we highlight this problem by giving new perspectives and try to improve the outcome of chemotherapy of cancer patients from different possible ways. Cancer metastases do not occur in all patients. $60 \%$ patients suffer those tumor types with low metastatic rates [18]. These patients will have good survival expectancy. To those patients, there is no need of additional anti-metastatic therapy. Only operations or therapy targeting primary tumors are enough. However, in many cases, we cannot know if a specific individual will metastasize or not? Or we have already found metastasized tumors when patients are diagnosed with cancer. There are two main types of options we can choose; (i) find out if some metastatic gene signatures in primary tumors [19-20]; (ii) treatment of metastatic foci with high active drugs. The central problem is ICC targeting tumor metastases. This needs to detect metastatic biomarkers in patients. If patients carry tumor species with high metastatic biomarkers, they need to be treated with anti-metastatic drugs.

\section{ICC: Are we Ready for that yet?}

Since, we may not possibly use all of these strategies in one patient, amongst different types of ICC, which of ICC strategy is the best? Each of them has its own advantages and disadvantages. At present, none of the ICC strategies are advantageous over the others. Also, no available ICC strategy has been well enough to significantly increase the patient's survival compared with conventional therapy. So we desperately need some magic moves to improve present ICC strategies or create new types of ICC strategies to integrate the advantages of all ICC types. Although much efforts have been made in fulfillment of ICC, but many main obstacles still need to be hurdled. For one important reason, there is almost no improvement in cancer patients' survival in spite of applications of ICC. But it can be a future miracle if we can make them perfect into a successful one. So are we ready for that yet? [21].

\section{References}

1. Lu da Y, Chen XL, Ding J (2006) Individualized cancer chemotherapy integrating drug sensitivity tests, pathological profile analysis and computational coordination-an effective strategy to improve clinical treatment. Med Hypotheses 66: 45-51.

2. Ocana A, Pandiella A (2010) Personalized therapies in the cancer "omics" era Mol Cancer 9: 202

3. Stransky B, Galante P. Application of bioinformatics in cancer research. An IMICS Perspective on Cancer Research 211-233.

4. Ichikawa W, Uetake H, Shirota Y, Yamada H, Nishi N, et al. (2003) Combination of dihydropyrimidine dehydrogenase and thymidylate synthase gene expressions in primary tumors as predictive parameters for the efficacy of fluoropyrimidine-based chemotherapy for metastatic colorectal cancer. Clin Cancer Res 9: 786-791.

5. Marx J (2004) Medicine. Why a new cancer drug works well, in some patients Science 304: 658-659.

6. Nowell PC (1976) The clonal evolution of tumor cell populations. Science 194 23-28.

7. Hanahan D, Weinberg RA (2000) The hallmarks of cancer. Cell 100: 57-70.

8. Stoler DL, Chen N, Basik M, Kahlenberg MS, Rodriguez-Bigas MA, et al. (1999) The onset and extent of genomic instability in sporadic colorectal tumor progression. Proc Natl Acad Sci USA 96: 15121-15126.

9. Jongeneel CV, Iseli C, Stevenson BJ, Riggins GJ, Lal A, et al. (2003) Comprehensive sampling of gene expression in human cell lines with massively parallel signature sequencing. Proc Natl Acad Sci USA 100: 4702-4705.

10. van de Vijver MJ, He YD, van't Veer LJ, Dai HY, Hart AM, et al. (2002) A geneexpression signature as a predictor of survival in breast cancer. $\mathrm{N}$ Engl J Med 347: 1999-2009

11. Gordon GJ, Jensen RV, Hsiao LL, Gullans SR, Blumenstock JE, et al. (2002) Translation of microarray data into clinically relevant cancer diagnostic tests using gene expression ratios in lung cancer and mesothelioma. Cancer Res 62: 4963-4967.

12. Diehl V (2007) Hodgkin's disease - from pathology specimen to cure. N Engl J Med 357: 1968-1971.

13. Geyer CE, Forster J, Lindquist D, Chan S, Romieu CG et al. (2006) Lapatinib plus capecitabine for HER2-positive advanced breast cancer. N Engl J Med 355: 2733-2743

14. Vermorken JB, Mesia R, Rivera F, Remenar E, Kawecki A, et al. (2008) Platinum-based chemotherapy plus cetuximab in head and neck cancer. $N$ Engl J Med 359: 1116-1127.

15. Younes A, Bartlett NL, Leonard JP, Kennedy DA, Lynch CM, et al. (2010) Brentuximab vedotin (SGM-35) for relapsed CD30-positive lymphomas. N Eng J Med 363: 1812-1821.

16. Tol J, Koopman M, Cats A, Rodenburg CJ, Creemers GJ, et al. (2009) Chemotherapy, bevacizumab, and cetuximab in metastatic colorectal cancer N Engl J Med 360: 563-572.

17. Miller K, Wang M, Gralow J, Dickler M, Cobleigh M, et al. (2007) Paclitaxel plus bevacizumab versus paclitaxel alone for metastatic breast cancer. $\mathrm{N}$ Engl $J$ Med 357: 2666-2676.

18. Siegel R, Ward E, Brawley O, Jamal A (2011) Cancer statistics, 2011: the impact of eliminating socioeconomic and racial disparities on premature cance deaths. CA Cancer J Clin 61: 212-236.

19. Valastyan S, Weinberg RA (2011) Tumor metastasis: molecular insights and evolving paradigms. Cell 147: 275-292.

20. Lu DY, Lu TR, Cao S (2012) Cancer metastases and clinical therapies. Cell Dev Biol 1: 1-3.

21. Lu DY, Lu TR, Chen XL (2012) Individualized cancer chemotherapy, are we ready for that yet? Metabolomics 2: 1 . 